\title{
Comparison of antiplatelet regimens in secondary stroke prevention: a nationwide cohort study
}

Christine Benn Christiansen ${ }^{1,2^{*}}$, Jannik Pallisgaard ${ }^{2}$, Thomas Alexander Gerds ${ }^{3}$, Jonas Bjerring Olesen², Mads Emil Jørgensen², Anna Karin Numé2, Nicholas Carlson², Søren Lund Kristensen², Gunnar Gislason 2,4,5 and Christian Torp-Pedersen ${ }^{6}$

\begin{abstract}
Background: In patients with ischemic stroke of non-cardioembolic origin, acetylsalicylic acid, clopidogrel, or a combination of acetylsalicylic acid and dipyridamole are recommended for the prevention of a recurrent stroke. The purpose of this study was to examine the risk of bleeding or recurrent stroke associated with these three treatments.

Methods: Patients who were discharged with first-time ischemic stroke from 2007-2010, with no history of atrial fibrillation were identified from Danish nationwide registries. Hazard ratios (HRs) and 1-year risks of recurrent ischemic stroke and bleeding were calculated for each antiplatelet regimen.

Results: Among patients discharged after first-time ischemic stroke, 3043 patients were treated with acetylsalicylic acid, 12,295 with a combination of acetylsalicylic acid and dipyridamole, and 3885 with clopidogrel. Adjusted HRs for clopidogrel versus the combination of acetylsalicylic acid and dipyridamole were 1.02 (95\% confidence interval [Cl]: 0.89-1.17) for ischemic stroke and 1.06 (95 \% Cl: 0.83-1.35) for bleeding. Adjusted HRs for acetylsalicylic acid versus the combination of acetylsalicylic acid and dipyridamole were 1.48 (95\% Cl: 1.31-1.67) for stroke and 1.47 (95\% Cl: 1.18-1.82) for bleeding. Clopidogrel versus acetylsalicylic acid yielded HRs of 0.69 (95\% Cl: 0.59-0.81) and 0.72 (95\% Cl: 0.55-0.96) for stroke and bleeding, respectively. The 1-year predicted risks associated with acetylsalicylic acid, the combination of acetylsalicylic acid and dipyridamole, and clopidogrel were 11.1 (95 \% Cl: 10.2-12.2), 7.7 (95 \% Cl: 7.3-8.3), and 8.0 (95\% Cl: 6.9-8.7) for ischemic stroke, respectively; while, the risks for bleeding were 3.4 (95\% Cl: 2.8-3.9), 2.4 (95\% Cl: 2.1-2.7), and 2.4 (95\% Cl: 1.9-2.9), respectively.

Conclusion: Clopidogrel and the combination of acetylsalicylic acid and dipyridamole were associated with similar risks for recurrent ischemic stroke and bleeding; whereas acetylsalicylic acid was associated with higher risks for both ischemic stroke and bleeding. The latter finding may partially be explained by selection bias.
\end{abstract}

Keywords: Ischemic stroke, Secondary prevention, Antiplatelet, Epidemiology

\footnotetext{
* Correspondence: christinebenn@hotmail.com

${ }^{1}$ Aalborg University Hospital, Forskningens Hus, Sdr. Skovvej 15, 9000

Aalborg, Denmark

2Department of Cardiology, Gentofte Hospital, Gentofte, Denmark

Full list of author information is available at the end of the article
} 


\section{Background}

After ischemic stroke or transient ischemia attack, patients are at high risk for a subsequent stroke. In the Unites States, $25 \%$ of the 795,000 annual ischemic strokes are recurrent events [1] and 70-84\% of ischemic stroke are non-cardioembolic [2, 3]. International guidelines recommend antiplatelets for secondary prevention in patients with strokes of non-cardioembolic origin [1, 4]. The recommended treatment regimens include acetylsalicylic acid, a combination of acetylsalicylic acid and dipyridamole, or clopidogrel.

Large-scale clinical randomized studies have evaluated the efficacy and safety of the recommended treatments. The combination of acetylsalicylic acid and dipyridamole was reported to be more effective than acetylsalicylic acid alone in preventing subsequent stroke, and may be associated with a decreased risk of bleeding $[5,6]$. In patients with ischemic stroke, no significant difference was found between clopidogrel and acetylsalicylic acid for the prevention of ischemic stroke/myocardial infarction (MI)/cardiovascular (CV) death [7]. Treatment with clopidogrel was as effective in preventing ischemic stroke as the combination of acetylsalicylic acid and dipyridamole, and was associated with a lower risk of bleeding [8]. Since the combination of acetylsalicylic acid and dipyridamole was associated with a lower risk of recurrent stroke than acetylsalicylic acid alone and clopidogrel was not significantly different from acetylsalicylic acid, it was surprising that there was no significant difference between clopidogrel and the combination of acetylsalicylic acid and dipyridamole. This paradox may be due to differences between study designs, such as inclusion criteria and drug dosage [9].

Epidemiological research in a real-world cohort can provide additional evidence of the efficacy and safety of antiplatelet regimens, and thereby help to guide future guidelines on therapy. Therefore, using data from Danish nationwide registries, we investigated the risk of recurrent stroke and bleeding in patients with ischemic stroke from January $1^{\text {st }} 2007$ to December $31^{\text {st }} 2010$. We compared patients treated with acetylsalicylic acid, the combination of acetylsalicylic acid and dipyridamole, and clopidogrel.

\section{Methods \\ Registries}

Data were accessed and linked through encrypted identification numbers using registries in Statistics Denmark. All Danish citizens carry a unique civil registration number and the registries cover the entire Danish population. Diagnoses were found in the National Patient Register, where diagnoses from hospital discharges are registered according to the International Classification of Diseases (ICD) with ICD-8 codes from 1978 and ICD-
10 codes from 1995 [10]. Information on medication was found in the Danish Medicinal Register, where filled prescriptions were registered since 1994 according to the Anatomical Therapeutic Chemical (ATC) Classification System [11]. High degree of validity for these data has been reported previously [12]. The cause and date of death was retrieved from the Danish Register of Causes of Death [13].

\section{Study population and antiplatelet therapy}

All patients discharged with first-time ischemic stroke (ICD-8: 433, 434, 436 or ICD-10: I63, I64) in the study period from January $1^{\text {st }} 2007$-December $31^{\text {st }} 2010$ were eligible for inclusion. Exclusion depended on the presence of the following before or up to 30 days after discharge: atrial fibrillation (ICD-8 code: 42793-42794, ICD-10: I48) at baseline or anticoagulation therapy (ATC: B01AA, B01AE07, B01AF02, B01AF01). Acetylsalicylic acid was defined by ATC-code B01AC06 or N02BA01 and clopidogrel was defined by ATC-code B01AC04. The combination of acetylsalicylic acid and dipyridamole was defined by either ATC-code B01AC30 or redeemed prescriptions for both acetylsalicylic acid and dipyridamole (ATC-code B01AC07). Patients that did not receive either acetylsalicylic acid, a combination of dipyridamole and acetylsalicylic acid, or clopidogrel at baseline were excluded. Acetylsalicylic acid may be sold as an over the counter drug in Denmark, in which case the purchase is not registered. However, prescription of the drug is required to obtain reimbursement, which enables linkage to an individual. Prescription is required for the acquisition of dipyridamole, clopidogrel, and glucose-lowering drugs.

\section{Comorbidities and concomitant medication}

Diabetes mellitus, heart failure, and renal disease were defined as previously [14, 15]: diabetes mellitus by the use of glucose-lowering drugs (ATC: A10) and heart failure (ICD-8: 425, 4270, 4271; ICD-10: I110, I42, I50, J819) and renal disease by diagnosis (ICD-8: 24902, 25002, 40399, 58000, 58199, 582-3, 59009, 591, 75234; ICD-10: E102, E112, E132, E142, I120, N02-8, N11-12, N14, N18-9, N26, N158-160, N162-4, N168, Q612-3, Q615, Q619). The discharge diagnosis prior to study inclusion was also used to define MI (ICD-8: 410; ICD-10: I21, I22), peripheral artery disease (ICD-8: 440; ICD-10 DI700, I702-9), chronic obstructive pulmonary disease (ICD-8: 491-2; ICD-10: J42-4), and cancer (ICD-8 140218 or ICD-10 codes C). Bleeding was defined as gastrointestinal bleeding (ICD-8: 53190, 53192, 53195, 53290, 53390, 53490, 53501, 56915; ICD-10: K250, K252 К254, K256, K260, K262, К264, K266, К270, К272, К274, K276, K920-2), intracranial hemorrhage, or bleeding from the urogenital or respiratory system (ICD-8: 
28001,43008-9,43098-9, 431; ICD-10 I60-62, N02, R04, R31, I690-2, J942) as done previously [15]. Hypertension was defined by concomitant use of medication from at least two of the following classes of antihypertensive drugs: antiadrenergics, diuretics, vasodilators, betablockers, calcium channel blockers, and renin angiotensin converting enzyme inhibitors at baseline. This definition of hypertension has been validated and has a specificity of $94.7 \%[14,16]$.

\section{Outcomes}

The following outcomes of interest were investigated: diagnoses of fatal or nonfatal ischemic stroke and fatal or nonfatal bleeding.

\section{Study design}

All patients with first-time ischemic stroke between January $1^{\text {st }} 2007$ and December $31^{\text {st }} 2010$ were identified. Data on outcomes until December $31^{\text {st }} 2011$ were available. Patients were included if they survived the first 30 days after stroke. Follow-up commenced 30 days after discharge to allow time to redeem prescriptions, and ended when patients experienced an outcome of interest, died, emigrated, or one year after discharge, whichever came first.

\section{Statistical analysis}

$P$-values for baseline characteristics were calculated with Chi-square test for categorical variables and Wilcoxon for continuous variables. HR of ischemic stroke and bleeding were calculated based on cause-specific Cox regression analyses. These analyses were adjusted for sex, age, year, prior myocardial infarction, hypertension, diabetes, peripheral artery disease, heart failure, chronic obstructive lung disease and cancer. The proportional hazard assumption was evaluated by score process tests [17].

The absolute risks of stroke (bleeding) within 1 year were calculated by combining the cause-specific Cox regression analysis for stroke (bleeding) with a second cause-specific Cox regression analysis for the competing risks. The latter was adjusted for the confounders listed above and also included the factual treatment. We predicted a personalized absolute 1-year risk of stroke (bleeding) for each patient in counter factual data were the treatment variable was set to acetylsalicylic acid (clopidogrel, acetylsalicylic acid/dipyridamole) for all patients but keeping the factual values of all confounders. Reported were mean 1-year risks of stroke (bleeding) across all patients associated with acetylsalicylic acid (clopidogrel, acetylsalicylic acid/dipyridamole) with bootstrap confidence limits (CI) based on 200 bootstrap samples drawn with replacement from the full data. All programming and analyses were run in SAS 9.4 (SAS Institute Inc., Gary, NC, USA) and R 3.0.2. ( $\mathrm{R}$ Core
Team (2013). R: A language and environment for statistical computing. (R Foundation for Statistical Computing, Vienna, Austria).

\section{Ethics}

Ethical approval is not required for historical registerbased studies in Denmark The study is consistent with the Declaration of Helsinki and was approved by the Danish Data Protection Agency (ref. 2007-58-0015, local journal number GEH 2014-013, I-suite number 02731). As the civil registration numbers of the patients were encrypted, individuals could not be identified. The authors take full responsibility for the integrity of data.

\section{Results}

\section{Study population}

A flowchart of the study population is shown in Fig. 1. Of the 42,295 patients included after a first-time ischemic stroke, 19,223 remained after exclusion of patients with atrial fibrillation, those taking oral anticoagulants, and patients not receiving any antithrombotic treatment. Of the included patients, 3043 were treated with acetylsalicylic acid, 12,295 with acetylsalicylic acid and dipyridamole, and 3885 with clopidogrel. Baseline characteristics are summarized in Table 1. Patients with acetylsalicylic acid alone were older (median age 75.3 years, interquartile range [IQR, 64.4-83.7]) and less often male (47.5\%) than patients treated with the combination of acetylsalicylic acid and dipyridamole (median age 70.7 years [IQR, 61.579.6], $56.5 \%$ males) or clopidogrel (median age 68.6 years [IQR, 59.2-77.6], $49.3 \%$ males). Patients receiving acetylsalicylic acid alone had more hypertension, renal disease, chronic obstructive pulmonary disease, and previous bleeding than patients treated with the combination of acetylsalicylic acid and dipyridamole or clopidogrel. Patients treated with the combination of acetylsalicylic acid and dipyridamole had less MIs then patients receiving acetylsalicylic acid and clopidogrel. The median follow-up time was 335 days [IQR, 335-335] for all treatment groups and the total follow-up time was $2475,10,601$, and 3364 person-years for acetylsalicylic acid, combination of acetylsalicylic acid and dipyridamole, and clopidogrel, respectively. The corresponding number of ischemic strokes was 360, 957 and 291 and the number of bleedings was 123, 286 and 92. Nine people emigrated; one person receiving clopidogrel, to receiving acetylsalicylic acid and six on combination therapy.

Time trends in antiplatelet regimens after ischemic stroke The proportion of patients with ischemic stroke assigned to the different antiplatelet regimens during the study period is shown in Fig. 2. From 2007 to 2010, the fraction of patients that received acetylsalicylic acid and the combination of acetylsalicylic acid and dipyridamole 


\section{Patients aged 30-100 identified with first ischemic stroke, 2007-2010 \\ 42,579}

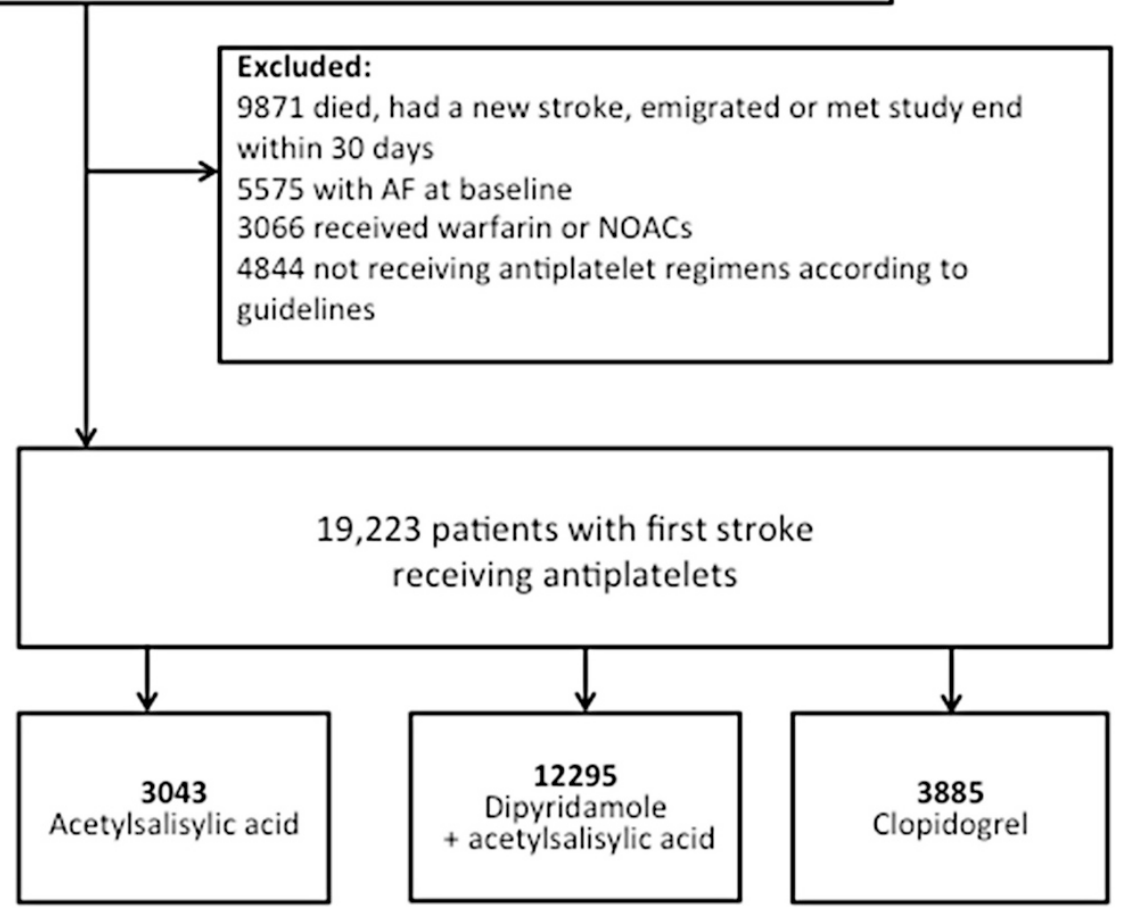

Fig. 1 Flowchart of study population

decreased from 19 to $12 \%$ and from 71 to $46 \%$, respectively; whereas, the proportion of patients that received clopidogrel increased from 10 to $41 \%$.

\section{Adherence to treatment}

The adherence to the antiplatelet regimen at baseline is shown in Table 2. The proportions of patients (alive and event free) that adhered to the baseline antiplatelet regimens were $86.2,91.3$, and $92.8 \% / 89.2 \%$ for acetylsalicylic acid, clopidogrel, and acetylsalicylic acid/dipyridamole, respectively.

\section{Risk of stroke and bleeding}

Adjusted HRs of ischemic stroke and bleeding are shown in Fig. 3. No significant difference was found for clopidogrel versus the combination of acetylsalicylic acid and

Table 1 Baseline characteristics

\begin{tabular}{|c|c|c|c|c|}
\hline & $\begin{array}{l}\text { Acetylsalicylic acid } \\
(n=3043)\end{array}$ & $\begin{array}{l}\text { Acetylsalicylic acid + dipyridamole } \\
(n=12,295)\end{array}$ & $\begin{array}{l}\text { Clopidogrel } \\
(n=3885)\end{array}$ & $p$-value \\
\hline Age in years, median (IQR) & $75.3(64.4-83.7)$ & $70.7(61.5-79.6)$ & $68.6(59.2-77.6)$ & $>0.0001$ \\
\hline Men (\%) & $47.5 \%$ & $56.5 \%$ & $49.3 \%$ & $>0.0001$ \\
\hline Diabetes & $380(12.5 \%)$ & 1449 (11.8\%) & $455(11.7 \%)$ & 0.5263 \\
\hline Hypertension & $1316(43.2 \%)$ & $4987(40.6 \%)$ & $1644(42.3 \%)$ & 0.0102 \\
\hline Myocardial infarction & $334(11 \%)$ & $824(6.7 \%)$ & $523(13.5 \%)$ & $<0.01$ \\
\hline Peripheral artery disease & $126(4.1 \%)$ & $297(2.4 \%)$ & $180(4.6 \%)$ & $<0.01$ \\
\hline Heart failure & $222(7.3 \%)$ & $496(4 \%)$ & $229(5.9 \%)$ & $<0.01$ \\
\hline Cancer & $206(6.8 \%)$ & $714(5.8 \%)$ & $245(6.3 \%)$ & 0.1062 \\
\hline Chronic obstructive pulmonary disease & $256(8.4 \%)$ & $808(6.6 \%)$ & $274(7.1 \%)$ & $<0.01$ \\
\hline Previous bleeding & $405(13.3 \%)$ & $853(6.9 \%)$ & 319 (8.2 \%) & $<0.01$ \\
\hline NSAIDS & 485 (15.9\%) & 1751 (14.2 \%) & 600 (15.4 \%) & 0.0243 \\
\hline
\end{tabular}




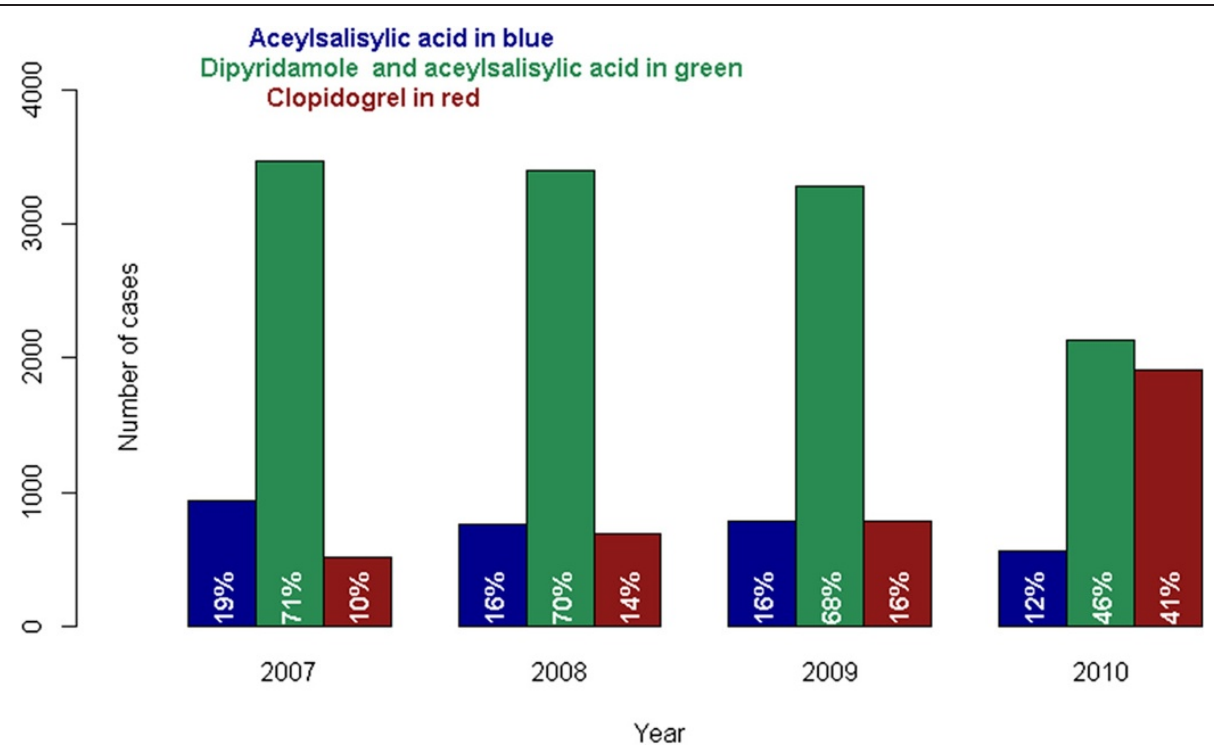

Fig. 2 Trends in treatments after first-time ischemic stroke from 2007 through 2010: Number of cases on each antiplatelet regimen

dipyridamole for stroke (HR 1.02 [95 \% CI: 0.89-1.17]) or bleeding (HR 1.06 [95 \% CI: 0.83-1.35]). Acetylsalicylic acid was associated with significantly increased HRs for both endpoints when compared with the combination of acetylsalicylic acid and dipyridamole, and clopidogrel was associated with decreased HRs for ischemic stroke and bleeding when compared with acetylsalicylic acid.

Absolute risks of ischemic stroke and bleeding are shown in Table 3. These results take into account the competing risk of death for reasons other than ischemic stroke and bleeding, and are associated with settings where all patients would be treated with acetylsalicylic acid (clopidogrel, acetylsalicylic acid/dipyridamole). At 3, 6, and 12 months, the risks of ischemic stroke and bleeding were similar for clopidogrel and combination of acetylsalicylic acid and dipyridamole. The acetylsalicylic acid group had generally high risks of bleeding and ischemic stroke.

\section{Discussion}

This historical register-based nationwide cohort study comprised more than 19,000 cases of first-time ischemic stroke without atrial fibrillation and treated with antiplatelet regimens. The risks of ischemic stroke and bleeding were similar for the combination of acetylsalicylic acid and dipyridamole and for clopidogrel; whereas, acetylsalicylic acid alone was associated with higher risks of both ischemic stroke and bleeding.

\section{Acetylsalicylic acid versus the combination of acetylsalicylic acid and dipyridamole}

The European Stroke Prevention Study 2 (ESPS 2, $n=$ 3299 with ischemic stroke or TIA within 3 months) found that the combination of acetylsalicylic acid and dipyridamole was more efficient than acetylsalicylic acid alone in preventing strokes. On the other hand, the European/Australasian Stroke Prevention in Reversible Ischemia Trial (ESPRIT) $(n=2739$ patients with stroke or TIA within 6 months) did not show significant reduction in the risk of ischemic stroke or all-cause death, but did find significantly decreased risk for the combined endpoint of ischemic stroke/MI/CV death with the combination therapy [5]. Of 1376 patients treated with the combination of acetylsalicylic acid and dipyridamole, 470

Table 2 Adherence to baseline drugs. Proportion of patients that redeemed prescriptions for each antiplatelet treatment at 6 and 12 months after first ischemic stroke

\begin{tabular}{lcc}
\hline Baseline treatment & $\begin{array}{c}\text { Proportion of patients that redeemed a } \\
\text { prescription after } 6 \text { months of baseline drug (\%) }\end{array}$ & $\begin{array}{c}\text { Proportion of patients that redeemed } \\
\text { a prescription after 12 months (\%) }\end{array}$ \\
\hline Clopidogrel & 91.3 & 87.5 \\
Acetylsalicylic acid and dipyridamole & 92.8 (acetylsalicylic acid) & 91.7 (acetylsalicylic acid) \\
& 89.2 (dipyridamole) & 87.3 (dipyridamole) \\
Acetylsalicylic acid & 86.0 & 85.6 \\
\hline
\end{tabular}




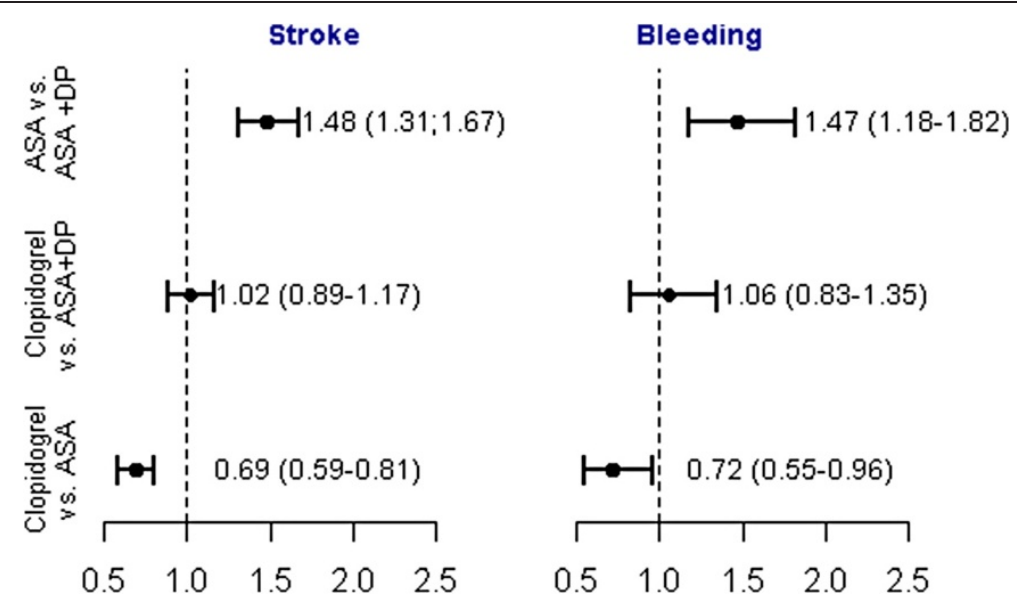

Fig. 3 Results from cause- specific Cox regression analysis: cause-specific hazard ratios for ischemic stroke and bleeding. Other death denotes any death other than cardiovascular death. Abbreviations: ASA = acetylsalicylic acid and DP= dipyridamole. All endpoints are adjusted for sex, age, prior myocardial infarction, hypertension, diabetes, peripheral artery disease, heart failure, chronic obstructive lung disease, and cancer. Bleeding is further adjusted for previous bleeding

patients discontinued treatment, compared with 184 in the acetylsalicylic acid group.

Bleeding was not an endpoint in ESPS 2, but similar proportions of bleeding were reported as adverse events in the acetylsalicylic acid and combination of acetylsalicylic acid and dipyridamole groups (135/1649 and 144/ 1650, respectively. ESPRIT reported a decreased risk of major bleeding (defined as intracranial bleeding, any fatal bleeding, or bleedings requiring hospital admission) for the combination of acetylsalicylic acid and dipyridamole in the on treatment group. The investigators explained this finding as a play of chance.

\section{Selection bias}

In an observational study, there is a risk of confounding by indication and selection bias since the study population is not randomized at baseline. In our study the risk of other death (other than fatal stroke) was increased in the acetylsalicylic acid group compared with the combination group, which indicates a difference between the two groups. As CV death overall and not just ischemic stroke may be influenced by antiplatelet treatment, we performed a sensitivity analysis of the combined endpoint of stroke/
MI/CV death (data not shown), where "other death" did not include MI or CV death and hence should not be influenced by antiplatelet treatment. However, also here the HR of "other death" remained increased for acetylsalicylic acid even after adjusting for comorbidity. This indicates that treatment with acetylsalicylic acid was associated with factors that led to poorer outcome and that this difference was not explained by the available data on comorbidity. Hence, all results regarding acetylsalicylic acid are likely negatively biased and should be interpreted with great care.

\section{Clopidogrel versus acetylsalicylic acid}

The Clopidogrel versus Aspirin in Patients at Risk of Ischemic Events (CAPRIE) study included patients with previous stroke, MI, or peripheral artery disease [7]. A significantly decreased risk of stroke/MI/CV death was reported for clopidogrel versus acetylsalicylic acid in the study population overall, but no decrease in risk was found for the sub-group analysis of patients included after ischemic stroke. In contrast to our findings, the higher risk of overall bleeding was not increased in CAPRIE. This discrepancy may be explained by selection

Table 3 Predicted 1-year risk of ischemic stroke and bleeding based on Cox regression analysis

\begin{tabular}{|c|c|c|c|c|c|c|}
\hline & \multicolumn{3}{|c|}{ Risk of ischemic stroke } & \multicolumn{3}{|c|}{ Risk of bleeding } \\
\hline & $\begin{array}{l}3 \text { months } \\
\text { after stroke }\end{array}$ & $\begin{array}{l}6 \text { months } \\
\text { after stroke }\end{array}$ & $\begin{array}{l}12 \text { months } \\
\text { after stroke }\end{array}$ & $\begin{array}{l}3 \text { months } \\
\text { after stroke }\end{array}$ & $\begin{array}{l}6 \text { months } \\
\text { after stroke }\end{array}$ & $\begin{array}{l}12 \text { months } \\
\text { after stroke }\end{array}$ \\
\hline Acetylsalicylic acid & $3.6[3.2 ; 4.0]$ & $6.9[6.3 ; 7.7]$ & $11.1[10.2 ; 12.2]$ & $1.2[0.9 ; 1.4]$ & $2.0[1.6 ; 2.4]$ & $3.4[2.8 ; 3.9]$ \\
\hline $\begin{array}{l}\text { Acetylsalicylic acid } \\
\text { and dipyridamole }\end{array}$ & $2.4[2.2 ; 2.7]$ & $4.7[4.4 ; 5.2]$ & $7.7[7.3 ; 8.3]$ & $0.8[0.7 ; 0.9]$ & $1.4[1.2 ; 1.6]$ & $2.4[2.1 ; 2.7]$ \\
\hline Clopidogrel & $2.5[2.2 ; 2.9]$ & $4.9[4.3 ; 5.4]$ & $8.0[6.9 ; 8.7]$ & $0.8[0.6 ; 1.0]$ & $1.4[1.0 ; 1.7]$ & $2.4[1.9 ; 2.9]$ \\
\hline
\end{tabular}

The model was adjusted for sex, age, year, prior myocardial infarction, hypertension, diabetes, peripheral artery disease, heart failure, chronic obstructive lung disease, and cancer 
bias, similar to what was described above for the combination of acetylsalicylic acid and dipyridamole versus acetylsalicylic acid.

\section{Clopidogrel versus acetylsalicylic acid and dipyridamole} The finding that clopidogrel is associated with a similar risk as the combination of acetylsalicylic acid and dipyridamole for ischemic stroke and the combined endpoint of stroke/MI/CV death is in accordance with results from the Prevention Regimen for Effectively avoiding Second Strokes (PRoFESS), where patients could be included up to 120 days after the qualifying stroke $(n=$ 20,332) [8]. PRoFESS reported no significant difference in the risk of hemorrhagic events, but did report increased risk of major bleeding (bleeding causing hospitalizations) in patients treated with the combination of acetylsalicylic acid and dipyridamole compared with clopidogrel recipients (HR 1.15 [95 \% CI: 1.00-1.32]). Differences in definitions of bleeding or statistical chance may also explain the difference in results. The network meta-analysis of ESPS2, ESPRIT, CAPRIE, and PRoFESS addressed the apparant paradox of clopidogrel being equal to the combination of acetylsalicylic acid and dipyridamole as well as to acetylsalicylic acid, when the combination of acetylsalicylic acid and dipyridamole was superior to acetylsalicylic acid alone. The analysis concluded that the risk of recurrence stroke was not significantly different between the three antiplatelet regimens [9].

\section{Strengths and limitations}

The study was a register-based observational study with built-in limitations that should be acknowledged. Due to the risk of selection bias, the most important limitation is that we do not have any data explaining the choice of antiplatelets, such as intolerance. Another important limitation is that we did not have access to data on stroke or bleeding severity. Minor bleedings remained unidentified in case they did not cause admissions to hospital. Minor bleedings remained unidentified in case they did not cause admissions to hospital. If the unregistered bleedings were equally distributed between treatment groups, the actual relative difference would be smaller, while the absolute difference would not be affected. In addition, we did not have data on the smoking status, body mass index, exercise, dosage of acetylsalicylic acid, or the diagnoses registered by general practitioners, and this may influence the results. The strengths include the size of the study with the advantage that data on hospitalizations and medication for the entire Danish population were available. The registration of the diagnosis of stroke has a high sensitivity and specificity [18].

\section{Generalizability}

This study is representative for the Danish population and results may plausibly apply to similar Western European countries. In other populations with different ages, ethnicities, socioeconomic and lifestyle factors, the stroke etiology may differ, which may affect the impact of antiplatelet therapy.

\section{Implications and further studies}

Our results indicate that clopidogrel or the combination of acetylsalicylic acid and dipyridamole are equal alternatives for the prevention of recurrent stroke in patients with ischemic stroke. As noted, due to the risk of selection bias, the results regarding acetylsalicylic acid should be interpreted with care.

\section{Conclusion}

In this nationwide study comprising more than 19,000 patients with ischemic stroke, the proportion of patients with first time ischemic stroke that received clopidogrel increased during the study period from 2007-2010. The risk of secondary ischemic stroke as well as the risk of bleeding were similar for treatment with clopidogrel and the combination of acetylsalicylic acid and dipyridamole. Treatment with acetylsalicylic acid alone was associated with worse outcomes, but was not comparable to the other groups due to risk of bias.

\section{Competing interests}

No conflicting relationship exists for any author.

\section{Authors' contributions}

All authors: critical revision of the manuscript, final approval of the document to be published, and agreement to be accountable for all aspects of the work in ensuring that questions related to the accuracy or integrity of any part of the work are appropriately investigated and resolved. CC: data collection, study design, interpretation of data, drafting of the manuscript and figures; TAG: study design, statistical analyses, and interpretation; JP and JBO: study design, data collection, and interpretation; NC, SLK, MEJ, AKN, and GG: data interpretation; CTP: study design, co-writing the manuscript, and revision of figures.

\section{Acknowledgements}

CC received a grant from The Danish Heart Foundation. The Danish Heart Foundation was not involved in the design, data collection, interpretation of data, the writing of the manuscript, or in the decision to submit the manuscript for publication.

$\mathrm{GG}$ received a grant and personal fees from AstraZeneca, grants from Bristol Meyers Squibb, and grants and personal fees from Pfizer and was also supported by an unrestricted clinical research scholarship from the Novo Nordisk Foundation. JBO has received speaker fees from Bristol-Myers Squibb and Boehringer Ingelheim and funding for research from the Lundbeck Foundation, Bristol-Myers Squibb, and The Capital Region of Denmark, Foundation for Health Research. CTP received grants from Pfizer, MSD, Sanofi, and Cardiome. AKN received a research grant from Helsefonden. JP has been supported by an unrestricted research grant from Boehringer-Ingelheim. MEJ, SLK and TAG report no funding related to this study. NC received a grant from the Danish Heart foundation unrelated to this study. None of the funding bodies played any role in the collection, analysis, or interpretation of data; in the writing of the manuscript; or the decision to submit the manuscript for publication. 


\section{Author details}

${ }^{1}$ Aalborg University Hospital, Forskningens Hus, Sdr. Skovvej 15, 9000

Aalborg, Denmark. ${ }^{2}$ Department of Cardiology, Gentofte Hospital, Gentofte, Denmark. ${ }^{3}$ Department of Public Health, Section of Biostatistics, University of Copenhagen, Copenhagen, Denmark. ${ }^{4}$ National Institute of Public Health, University of Southern Denmark, Copenhagen, Denmark. ${ }^{5}$ Faculty of Health and Medical Sciences, University of Copenhagen, Copenhagen, Denmark. ${ }^{6}$ Department of Health Science and Technology, Aalborg University, Aalborg, Denmark.

Received: 5 February 2015 Accepted: 22 October 2015

Published online: 02 November 2015

\section{References}

1. Furie KL, Kasner SE, Adams RJ, Albers GW, Bush RL, Fagan SC, et al. Guidelines for the prevention of stroke in patients with stroke or transient ischemic attack: a guideline for healthcare professionals from the American heart Association/American Stroke Association. Stroke. 2011;42(1):227-76. doi:10.1161/STR.0b013e3181f7d043.

2. Markus HS, Khan U, Birns J, Evans A, Kalra L, Rudd AG, et al. Differences in stroke subtypes between black and white patients with stroke: the South London Ethnicity and Stroke Study. Circulation. 2007;1 16(19):2157-64. doi:10.1161/CIRCULATIONAHA.107.699785.

3. Troster S, Schuster HP, Bodmann KF. Atrial fibrillation in patients of a medical clinic-a marker for multi-morbidity and unfavorable prognosis. Med Klin. 1991;86(7):338-43.

4. European Stroke Organisation Executive C, Committee ESOW. Guidelines for management of ischaemic stroke and transient ischaemic attack 2008. Cerebrovasc Dis. 2008;25(5):457-507. doi:10.1159/000131083.

5. Halkes PH, van Gijn J, Kappelle LJ, Koudstaal PJ, Algra A. Aspirin plus dipyridamole versus aspirin alone after cerebral ischaemia of arterial origin (ESPRIT): randomised controlled trial. Lancet. 2006;367(9523):1665-73. doi:10.1016/S0140-6736(06)68734-5.

6. Diener HC, Cunha L, Forbes C, Sivenius J, Smets P, Lowenthal A. European Stroke Prevention Study. 2. Dipyridamole and acetylsalicylic acid in the secondary prevention of stroke. J Neurol Sci. 1996;143(1-2):1-13.

7. CAPRIE Steering Committee. A randomised, blinded, trial of clopidogrel versus aspirin in patients at risk of ischaemic events (CAPRIE). Lancet. 1996;348(9038):1329-39.

8. Sacco RL, Diener HC, Yusuf S, Cotton D, Ounpuu S, Lawton WA, et al. Aspirin and extended-release dipyridamole versus clopidogrel for recurrent stroke. N Engl J Med. 2008;359(12):1238-51. doi:10.1056/NEJMoa0805002.

9. Kent $D M$, Thaler DE. Stroke prevention-insights from incoherence. N Engl J Med. 2008:359(12):1287-9. doi:10.1056/NEJMe0806806.

10. Lynge E, Sandegaard JL, Rebolj M. The Danish national patient register. Scand J Public Health. 201 1;39(7 Suppl):30-3. doi:10.1177/1403494811401482.

11. Kildemoes HW, Sorensen HT, Hallas J. The Danish national prescription registry. Scand J Public Health. 2011:39(7 Suppl):38-41. doi:10.1177/1403494810394717.

12. Gaist D, Sorensen HT, Hallas J. The Danish prescription registries. Dan Med Bull. 1997:44(4):445-8.

13. Helweg-Larsen K. The Danish register of causes of death. Scand J Public Health. 2011;39(7 Suppl):26-9. doi:10.1177/1403494811399958.

14. Ekholm O, Hesse U, Davidsen M, Kjoller M. The study design and characteristics of the Danish national health interview surveys. Scand J Public Health. 2009:37(7):758-65. doi:10.1177/1403494809341095.

15. Olesen JB, Lip GY, Kamper AL, Hommel K, Kober L, Lane DA, et al. Stroke and bleeding in atrial fibrillation with chronic kidney disease. N Engl I Med. 2012;367(7):625-35. doi:10.1056/NEJMoa1 105594.

16. Olesen JB, Lip GY, Hansen ML, Hansen PR, Tolstrup JS, Lindhardsen J, et al. Validation of risk stratification schemes for predicting stroke and thromboembolism in patients with atrial fibrillation: nationwide cohort study. BMJ. 2011;342:d124. doi:10.1136/bmj.d124.

17. Torben Martinussen THS. Dynamic regression models for survival data. New York: Springer; 2006.

18. Krarup LH, Boysen $G$, Janjua $H$, Prescott E, Truelsen T. Validity of stroke diagnoses in a national register of patients. Neuroepidemiology. 2007;28(3):150-4. doi:10.1159/000102143.

\section{Submit your next manuscript to BioMed Central and take full advantage of:}

- Convenient online submission

- Thorough peer review

- No space constraints or color figure charges

- Immediate publication on acceptance

- Inclusion in PubMed, CAS, Scopus and Google Scholar

- Research which is freely available for redistribution

Submit your manuscript at www.biomedcentral.com/submit 